\title{
Antimicrobial and Antioxidant Activity of Phenolic Compounds Extracted from Wine Industry By-Products of Tinto Cão Variety
}

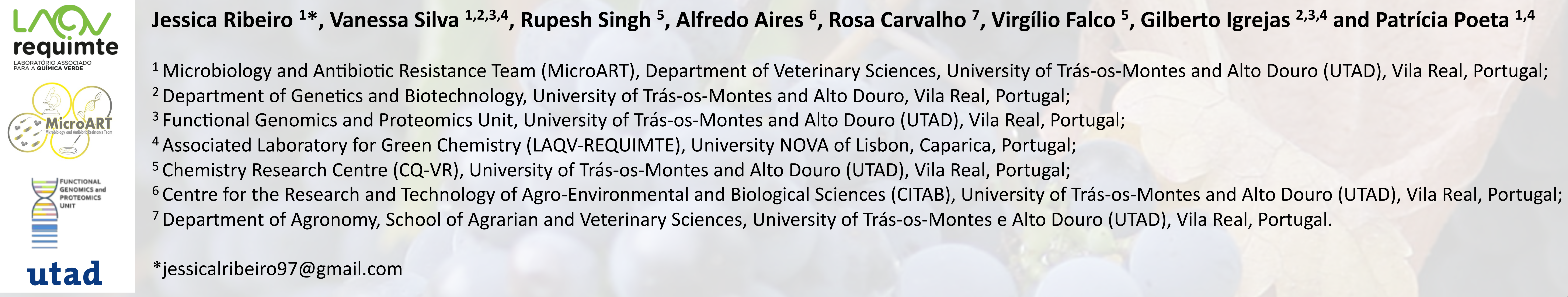

\section{Introduction}

In Portugal, wine production is one of the most important and relevant socio-economic activities. Portugal comprises a total of 14 wine regions which includes the Douro Demarcated Region. This region is the oldest and one of the most important wine regions of the country [1]. "Tinto Cão" (Vitis vinifera), which is a very important variety for Port wine production, is one of the most widely grown in the Douro Demarcated Region.

One litre of produced wine generates between 1.3 and $1.5 \mathrm{~kg}$ of waste: $75 \%$ is wastewater and the rest is organic waste, wine lees and inorganic waste. This waste is frequently regarded as an environmental issue, due to its negative impact when disposed. The quality of grape-derived products depends on the levels of secondary metabolites in the berries. In particular, grapes are rich in polyphenols. These compounds exhibit a wide range of physiological properties: antioxidant, anti-mutagenic, anti-inflammatory, anti-carcinogenic and antimicrobial activity [2].

The failure of many antibiotics in the treatment of infections caused by multi-drug resistant bacteria reenforces the necessity of effective strategies to combat this global healthcare issue [3]. Several studies showed that natural plant-derived compounds could be an alternative to combat bacteria that are resistant to conventional antibiotics [4]. Therefore, the aim of this study was to extract phenolic compounds from winery by-products (grape skin, seeds, stem, shoat and leaves) from Tinto Cão variety and evaluated their antioxidant activity and antibacterial properties against antibiotic resistant bacterial strains.

\section{Material and Methods}

The polyphenolic constituents were extracted using water/ethanol $(20: 80)$ mixture. The extracts were dissolved in dimethyl sulfoxide (DMSO) to a final concentration of $100 \mathrm{mg} / \mathrm{mL}$. Antimicrobial susceptibility assay was performed using Kirby-Bauer disc diffusion method and was tested against ten different bacteria: Listeria monocytogenes, Bacillus cereus, Staphylococcus aureus, Staphylococcus epidermidis, Enterecoccus faecium, Enterococcus faecalis, Klebsiella pneumoniae, Pseudomonas aeruginosa, Salmonella enteritidis and Escherichia coli. The antioxidant activity of the extracts was determined by DPPH, FRAP and CUPRAC methods.

\section{Results}

There was no inhibitory effect of neither of the extracts on $E$. faecium, E. faecalis, S. enteritidis and E. coli at the concentrations tested (Table 1). Among all bacteria tested, S. epidermidis was the most susceptible, followed by $K$. pneumoniae and $L$. monocytogenes. The shoat extracts showed the highest antimicrobial activity, as evidenced by the inhibitory effect on 6 out of the 10 bacterial strains tested and by lower MIC values.

Table 1. Minimum inhibitory concentration (MIC, $\mathrm{mg} / \mathrm{mL}$ ) and inhibition zones $(\mathrm{mm})$ of the phenolic extracts Tinto Cão grape skin, seeds, stem, shoat and leaves against multidrug resistant Gram-positive and Gram-negative bacteria.

\begin{tabular}{|c|c|c|c|c|c|}
\hline \multirow{2}{*}{ Bacterial Strain } & \multicolumn{5}{|c|}{ MIC (mg/mL) (Inhibition zone $(\mathrm{mm}))$} \\
\hline & Skin & Seed & Stem & Shoat & Leaf \\
\hline L. monocytogenes & - & $50(10)$ & $50(10)$ & $25(9)$ & $100(10)$ \\
\hline B. cereus & - & - & $25(9)$ & $50(10)$ & - \\
\hline E. faecium & - & - & - & - & - \\
\hline E. faecalis & - & - & - & - & - \\
\hline S. aureus & - & $75(10)$ & $25(8)$ & $10(8)$ & - \\
\hline S. epidermidis & $100(10)$ & $75(9)$ & $25(10)$ & $25(10)$ & $50(10)$ \\
\hline P. aeruginosa & - & - & $50(9)$ & $25(10)$ & - \\
\hline K. pneumoniae & - & $50(10)$ & $25(9)$ & $25(9)$ & $75(10)$ \\
\hline S. enteritidis & - & - & - & - & - \\
\hline E. coli & - & - & - & - & - \\
\hline
\end{tabular}

Results from all assays are expressed in effective concentration (EC50), and the lower the value the higher is the antioxidant power. The results obtained are shown in Table 2 , and according to it, seed extracts had the highest antioxidant activity with the DPPH and the CUPRAC method and the leaf had the highest antioxidant activity with the FRAP method.

Table 2. Antioxidant activity of the grape skin, seeds, stem, shoat and leaves of Tinto Cão (mean value \pm SD, $n=3$ ).

\begin{tabular}{|c|c|c|c|}
\hline $\begin{array}{c}\text { Tinto Cão } \\
\text { Components }\end{array}$ & \multicolumn{3}{|c|}{ Method } \\
\cline { 2 - 4 } Skin & DPPH & FRAP & CuPRAC \\
Seed & $1,81 \pm 0,09$ & $0,573 \pm 0,008$ & $0,541 \pm 0,002$ \\
Stem & $1,33 \pm 0,04 \pm 0,02$ & $0,573 \pm 0,002$ & $0,515 \pm 0,002$ \\
Shoat & $4,16 \pm 0,27$ & $0,584 \pm 0,007$ & $0,536 \pm 0,005$ \\
Leaf & $0,97 \pm 0,03$ & $0,548 \pm 0,001$ & $0,541 \pm 0,003$ \\
\hline
\end{tabular}

\section{Conclusion}

These results demonstrate that, natural products, such as polyphenols, may represent a promising mean to fight microbial resistance. However, they were not effective against all bacteria which indicates that these compounds, alone, might not substitute antibiotics. In addition, the extracts were effective against foodborne bacteria which shows that polyphenols may have potential usefulness as food preservers. Thus, the phenolic compounds may be used in food and pharmaceutical industries as natural food preservers and antibiotic adjuvants.

\section{ACKNOWLEDGMENTS:}

This work was also supported by the Associate Laboratory for Green Chemistry-LAQV which is financed by national funds from FCT/MCTES (UID/QUI/50006/2020). Vanessa Silva is grateful to FCT (Fundação para a Ciência e a Tecnologia) for financial support through PhD grant SFRH/BD/137947/2018. 